\title{
Fast Data Acquisition and Database Construction Scheme of Urban Traffic Information System Based on CORS
}

\author{
Bi He ${ }^{1,{ }^{*}}$, Baoqun Wang ${ }^{1}$ and Lili Tao ${ }^{2}$ \\ ${ }^{1}$ School of Civil Engineering, Shandong Jiaotong University, Jinan 250357, China \\ ${ }^{2}$ School of Automotive Engineering, Shandong Jiaotong University, Jinan 250357, China \\ * hebiy@163.com
}

\begin{abstract}
Traffic system is a complicated system, to realize digital management of transportation system, it is necessary to obtain the digital information of all component, and build the database to save and organize the digital information. According to the distribution characteristics of traffic system, CORS is a good choice to finish this work. In this paper, the method of using CORS technology to acquisition digital data of traffic system is introduced detailed, and the method of building the database is provided at the same time, these can be a beneficial reference for construction of traffic information system.
\end{abstract}

Keywords: Traffic system; CORS; digital management; digital data.

\section{Introduction}

Digital management is an effective method for improving the efficiency of the transportation system, and the basic digital management method is establishing the digital traffic information system. The foundation of this digital system is acquisition data of each component of traffic system and build a database to save and organize the data. The data mainly include spatial data and property data, spatial data is more difficultly to get, because it needs people to work in field. In practice, using CORS technology can rapid, accurate and low cost to finish the work.

\section{Principle of CORS}

CORS(Continuous Operational Reference System) is a fast accurate measurement technology, it refers to place GPS measurement instrument on a coordinate precisely known point, based on the precise coordinate and measurements by GPS, the coordinate error(corrected value) of the point can be gained, and then transfer the corrected value to the rest unknown point, based on the measurements and the corrected value, the precise coordinate of the rest point can be gained. The principle can also use the formula to express like this:

$$
\begin{aligned}
& X_{P 1}^{-}={\tilde{X_{P 1}}}^{-} X_{P 1} ; \bar{Y}_{P 1}=\tilde{Y_{P 1}}-Y_{P 1} \\
& X_{P 2} \cong \tilde{X_{P 2}}-X_{P 1}^{-} ; Y_{P 2} \cong \tilde{Y_{P 2}}-Y_{P 1}^{-}
\end{aligned}
$$

\section{The Advantages of Using CORS for Data Acquisition}

The main advantages of using CORS for data acquisition include two facts: fast speed and high accuracy.

The traditional methods such as total station measurement is indirect measurement, it means the measuring instrument is need to set up on the known points, by measuring the relative position of known and unknown points relationship to calculate the coordinates data of unknown points, in this process, set up equipment, data calculation is very complicated, which cause the overall operation speed being slow. At the same time, subject to the instrument facts, all-weather work cannot be realized, it also affects the operation speed. CORS is direct measurement, it means the measuring instrument is set up on the unknown points, the coordinate data of unknown points was gained directly, 
and CORS instrument is not affected by Factors such as environment and weather, all-weather work can be realized, so the operation speed can be Accelerated significantly.

Basic tools of CORS is still GPS, but one disadvantage of GPS is the measurement accuracy is less than some traditional ways such like total station, if civilian GPS not through the elaboration late processing, single point error can reach about 20 to $30 \mathrm{~m}$, it cannot meet the accuracy requirement of traffic information system. But CORS improved this problem, by introducing a corrected value from known point to fix observed value of unknown points, greatly improve the accuracy, the accuracy of final results can satisfy the requirements of traffic information system completely.

\section{Method of using CORS Operations}

The first step of using CORS to operate is select the datum point, it is a known point, which means the point coordinate is known and accurate, set up a GPS instrument on this datum point, the observed coordinate value can be gained, based on the accurate coordinate and the observed coordinate, the corrected value can be calculated, then save the corrected value. In this step, the importance is selection of datum point location, the datum point location cannot far away from the whole survey area, if the datum point location far away from the whole survey area, the corrected value which gained from the datum point maybe unfit for some unknown points in survey area.

The second step of using CORS to operate is transfer the corrected value to unknown points, GSM communication networks can be used to finish this work, with GSM, an internal communication network can be established, this network connects the GPS instruments on datum point and known points, transfer corrected value and other information continually each other.

The third step of using CORS to operate is survey the unknown points coordinate, set up GPS instrument on the unknown points, then the observed value can be gained, at the same time, received the corrected value which transfer from datum point through GSM, based on the observed value and corrected value, the relatively accurate coordinate value of unknown points can be gained.

\section{Method of Building Database}

Cause the traffic information system requires the data was organized and standard, one database is need to established to save, manage, organize and use the data which was acquisition by CORS.

According to the current application reality, establish a relational database is a good choice. Relational database use two-dimensional table to store the data, we can store the name of points in the columns and coordinate value in the rows, it can be expressed like Figure1:

\begin{tabular}{|c|c|c|c|}
\hline & $C(\mathrm{X})$ & $C(\mathrm{Y})$ & $C(\mathrm{Z})$ \\
\hline $\mathrm{P} 1006$ & 1124.32 & 265.38 & 122.86 \\
\hline $\mathrm{P} 1007$ & & & \\
\hline $\mathrm{P} 1008$ & $\ldots \ldots$ & ( & \\
\hline P1009 & $\ldots \ldots$ & & \\
\hline $\mathrm{P} 1010$ & $\ldots \ldots$ & & \\
\hline P1011 & ㄱ. & ... & \\
\hline $\mathrm{P} 1012$ & $\ldots \ldots$ & $\cdots$ & \\
\hline $\mathrm{P} 1013$ & & & \\
\hline P1014 & $\ldots \ldots$ & & \\
\hline P1015 & & & \\
\hline $\mathrm{P} 1016$ & $\ldots$ & & $\ldots$ \\
\hline P1017 & & & \\
\hline $\mathrm{P} 1018$ & 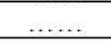 & & \\
\hline P1019 & & & \\
\hline $\mathrm{P} 1020$ & & & \\
\hline $\mathrm{P} 1021$ & 1966.62 & 598.26 & 134.77 \\
\hline
\end{tabular}

Figure 1. Coordinate value store in table

Commonly used relational database tools all can be used to finish the work, such like Access, Visual FoxPro, SQL Server, Oracle, etc. 


\section{Link to Traffic Information System}

Traffic information system is generally consisting of three parts: data layer, platform layer and application layer. The data layer's mission is to store and use data, platform layer's mission is to supply the running of whole system, the application layer's mission is running specific application program. link the database to traffic information system is the foundation for whole system running successfully. A kind of simple and quick method is to write a data interface in the system, through the data interface, the platform layer can directly read data in the database, and the data will be stored in the storage unit of the platform layer, and then provide for the use of the application layer, at the end, the use results will be sent back to the data layer.

\section{Conclusion}

The introduction of traffic information system is effective measures to improve the traffic management level and efficiency, and basic data acquisition and use is the key to success of the traffic information system. With the characteristics of fast speed and high accuracy, CORS is a good choice for the data acquisition of traffic information system, use CORS to gather coordinate data, and build the database to store the data, can greatly improve the construction speed of traffic information system, and can effectively guarantee the quality of the system, and This method will soon become the mainstream.

\section{Acknowledgments}

In this paper, the research was sponsored by Shandong Jiaotong University Science Research Foundation (Project No.201613).

\section{References}

[1]. Junyong Chen, Yamin Dang. On the progress in GNSS and Construction of its CORS System in China[J]. Geo-spatial Information, (2009) 7 (3) 1-3.

[2]. Weiping Jiang, Xuan Zou, Weiming Tang. A new kind of real-time PPP method for GPS singlefrequency receiver using CORS network[J]. Chinese Journal of Geophysics, (2012) 5 (5) 15491556.

[3]. Zhongxin Chen, Liqiang Zhu. Creating the virtual original observations of high accuracy GPS data based on CORS[J]. Science of Surveying and Mapping, (2011) 36 (1) 17-19.

[4]. Yuhua Huang, Hongyu Ni. Study on Technology for CORS Service[J]. Geo-spatial Information, (2008) 6(1) 34-36.

[5]. Xing Chen, Pengfei Cheng, Yingyan Cheng, Wentao Fan, Xiaoming Wang, Fuli Wang. Model of Crustal Vertical Movement Velocity Field Based on Hebei CORS Network[J]. Journal of Geodesy and Geodynamics, (2013) 33(4) 48-51.

[6]. Zhaosheng Nie, Yong Huang, Gang Liu, Zhige Jia. Quality evolution of Data from CORS in Hubei[J]. Journal of Geodesy and Geodynamics, (2013) 33(6) 53-51.6

[7]. Shuangcheng Zhang, Haiyang Cao, Han Gao, Haiying Li. Application and analysis of GPS Short Baseline Processing Based on GAMIT[J]. Survey and Mapping Bulletin, (2011) (10) 27-29.

[8]. Xi Li, Wusheng Hu, Hongfei Sun. Forest highway survey and empirical analysis based on CORS[J]. Journal of Southeast University (Natural Science Edition), (2013) (11) 360-363.

[9]. Xuan Zou, Weiming Tang, Chuang Shi, Jingnan Liu. Instantaneous Ambiguity Resolution For PPP-RTK Using Regional Ground-Based Augmentation Information[J], (2014) 34(1) 78-83. 
[10]. Weiping Jiang, Peng Yuan, Zhi Tian, Yugang Xiao. Coordinate Datum Unification for CORS network Combination[J], (2014) 39(5) 566-570. 